\title{
BMJ Open Identifying harm reduction strategies for alcohol and drug-use in inpatient care settings and emergency departments: a scoping review protocol
}

\author{
Janet A Curran (D) , ${ }^{1,2}$ Mari Somerville, ${ }^{1}$ Leah Boulos, ${ }^{3}$ Alexander Caudarella, ${ }^{4}$ \\ Daniel Crowther (D) , ${ }^{1}$ Catie Johnson (D) , ${ }^{2}$ Lori Wozney, ${ }^{5}$ Shannon MacPhee, ${ }^{2}$ \\ Douglas Sinclair, ${ }^{2}$ Annette Elliott Rose, ${ }^{2}$ Caroline Jose, ${ }^{3}$ Morgan Joudrey ${ }^{2}$
}

To cite: Curran JA,

Somerville M, Boulos L, et al. Identifying harm reduction strategies for alcohol and drug-use in inpatient care settings and emergency departments: a scoping review protocol. BMJ Open 2021;11:e055654. doi:10.1136/ bmjopen-2021-055654

- Prepublication history and additional supplemental material for this paper are available online. To view these files, please visit the journal online (http://dx.doi.org/10.1136/ bmjopen-2021-055654).

Received 19 July 2021 Accepted 28 September 2021

Check for updates

(c) Author(s) (or their employer(s)) 2021. Re-use permitted under CC BY-NC. No commercial re-use. See rights and permissions. Published by BMJ.

${ }^{1}$ School of Nursing, Dalhousie University, Halifax, Nova Scotia, Canada

${ }^{2}$ Quality and Patient Safety, IWK Health Centre, Halifax, Nova Scotia, Canada

${ }^{3}$ Maritime SPOR Support Unit, Halifax, Nova Scotia, Canada ${ }^{4}$ Mental Health and Addictions Service, St Michael's Hospital, Toronto, Ontario, Canada ${ }^{5}$ Mental Health and Addictions Program, Nova Scotia Health Authority, Halifax, Nova Scotia, Canada

Correspondence to

Janet A Curran; jacurran@dal.ca

\section{ABSTRACT}

Introduction People who use alcohol and/or drugs (PWUAD) are at high risk of medical complications, frequent hospitalisation and drug-related death following discharge from inpatient settings and emergency departments (EDs). Harm reduction strategies implemented in these settings may mitigate negative health outcomes for PWUAD. However, the scope of harm reduction strategies used globally within inpatient settings and EDs is unknown. The objective of this review is to identify and synthesise reported harm reduction strategies that have been implemented across inpatient settings and EDs for PWUAD.

Methods and analysis This review will include studies from any country and health service reporting on harm reduction strategies implemented in inpatient settings or EDs. The population of interest includes people of any race, gender and age identifying as PWUAD, or individuals who provided care to PWUAD. Studies which describe implementation strategies and barriers and enablers to implementation will be included. Studies published in English, or those available for English translation will be included. The following databases will be searched: MEDLINE All (Ovid), Embase (Elsevier Embase.com), CINAHL with Full Text (EBSCOhost), PsycINF0 (EBSCOhost) and SCOPUS (Elsevier Scopus.com). A grey literature search will be conducted. There will be no date restrictions on the search. Titles, abstracts and full texts will be screened in duplicate. Data will be extracted using a standardised form. The results will be reported using the Preferred Reporting Items for Systematic Reviews and Meta-analyses extension for scoping reviews.

Ethics and dissemination Scoping reviews do not require ethical approval. Patient partners with lived experience and relevant knowledge users will be engaged as research team members throughout all phases of the research process. A report detailing context, methodology and findings from this review will be disseminated to knowledge users and relevant community stakeholders. This review will be submitted for publication to a relevant peer-reviewed journal.

\section{INTRODUCTION}

Alcohol and drug use is associated with significant negative health outcomes. People who
Strengths and limitations of this study

- To the best of our knowledge, this scoping review will be the first of its kind to describe the range of harm reduction strategies for people who use alcohol and/or drugs that have been implemented in inpatient and emergency department settings.

- This scoping review will be conducted in accordance with the Joanna Briggs Institute methodology for scoping reviews and will take an integrated knowledge translation approach by engaging key knowledge users and patient partners throughout the research process.

- Our search will be limited to five databases (MEDLINE All (Ovid), Embase (Elsevier Embase.com), CINAHL with Full Text (EBSCOhost), PsycINF0 (EBSCOhost) and SCOPUS (Elsevier Scopus.com)) and searches for unpublished studies and grey literature will be retrieved and included following the systematic approach to grey literature searching outlined by Godin et al.

- Given that this is a scoping review, it will not contain a meta-analysis of available evidence and will only describe how research in this field is being conducted, the types of evidence that are being produced and specific knowledge gaps within the literature.

- Our search will be limited to English literature and non-English literature that is available for translation.

use alcohol and/or drugs (PWUAD) have a higher risk of infectious disease, drugrelated complications and are more likely to be frequently hospitalised when compared with other individuals. ${ }^{12}$ When seeking care, PWUAD are often discharged against medical advice or expelled as a result of illicit drug use, leading to increased readmission rates and poorer hospital outcomes. ${ }^{2}$ Encountering stigma or having negative experiences in hospital settings can prevent PWUAD from seeking medical help. ${ }^{3}$ Furthermore, PWUAD have a high risk of drug-related 
death after being discharged from hospital. ${ }^{4}$ Therefore, PWUAD face health-related risks before, during and after receiving medical care, highlighting an urgent need to improve healthcare.

Harm reduction is a pragmatic approach geared towards addressing immediate needs across a wide range of health issues. Harm reduction aims to improve the health, safety and well-being of both the individual and the community. ${ }^{56}$ Within the context of drug and alcohol use, harm reduction strategies provide alternatives to drug and alcohol abstinence for individuals who cannot or do not want to stop using. ${ }^{7-9}$ The Canadian Drugs and Substances Strategy has identified harm reduction as an integral part of their strategy to help address some of the burden associated with substance use. ${ }^{9}$ Some of the most common harm reduction strategies used in the context of illegal substances include clean needle distribution, supervised drug intake, substitution therapy, safe supply and the use of substances like naloxone to temporarily reverse the effects of opioid overdose.$^{7-11}$ These strategies aim to improve well-being, lower rates of illness, overdose and death because of substance use. ${ }^{6-9}$ Harm reduction in relation to substance use has been implemented in community-based, ${ }^{12}$ home-based ${ }^{13}$ and inpatient settings. ${ }^{14}$ However, there is a need to further understand the range of these harm reduction strategies.

In inpatient settings and emergency departments (EDs), there is some indication that harm reduction strategies could help to improve health outcomes for PWUAD. ${ }^{15-17}$ The use of these strategies within inpatient settings and EDs, remains low, despite high numbers of patients hospitalised with substance-induced symptoms ${ }^{18}$ and calls for harm reduction implementation in hospital settings. ${ }^{19}$ Low uptake of harm reduction strategies has been attributed to inadequate staffing, lack of funding and stigma surrounding substance use. ${ }^{20}$ Yet, to the best of our knowledge, the full range of available implementation strategies used in implementing harm reduction strategies and the range of barriers and enablers to implementation has not been adequately described.

A preliminary search of MEDLINE, the Cochrane Database of Systematic Reviews and JBI Evidence Synthesis was conducted and no current or underway systematic or scoping reviews on this topic were identified. Therefore, this study aims to identify and synthesise the literature on harm reduction strategies that have been implemented in inpatient settings and EDs among people who use substances.

\section{Review questions}

The following questions will guide this review:

1. What harm reduction strategies have been evaluated to help alleviate negative health outcomes associated with substance use within inpatient settings and EDs?

2. How are harm reduction strategies implemented in inpatient settings and EDs?

3. What are the reported barriers and enablers to their implementation?
4. What are the commonly reported outcome measures used to evaluate harm reduction strategies and their implementation in these settings?

\section{METHODS AND ANALYSIS}

The proposed scoping review will be conducted in accordance with the JBI methodology for scoping reviews. ${ }^{21}$ This scoping review will use an integrated knowledge translation approach, working with health system decision makers and patient partners with lived experience through all stages of the review. ${ }^{22}$ This scoping review has been registered with The Open Science Framework (OSF) registries (Registration DOI: 10.17605/OSF.IO/ P7BHN).

\section{Inclusion criteria \\ Participants}

This review will consider studies that include participants identifying as individuals with drug dependence or PWUAD who have been admitted to inpatient care or have accessed EDs for substance use related issues and/ or any other medical issues. This review will also consider individuals who provide care for persons with drug dependence or PWUAD. People of any race, gender and age will be included.

\section{Concept}

This review will consider studies that investigate any intervention or implementation strategy of an intervention designed to reduce harm related to negative health outcomes associated with alcohol and/or drug use. Descriptions of implementation strategies may include any barriers and/or enablers to facilitating harm reduction strategies. This review will consider reported outcome measures, and not outcome data. These will include patient reported outcome measures (eg, quality of life), and patient reported experience measures (eg, feeling heard, receiving care asked for), as well as health outcome measures (eg, length of stay, healthcare costs, recovery time, discharge against medical advice, readmission rates, overdose rates and mortality).

\section{Context}

Any healthcare setting that provides inpatient care or has an ED, in any country will be considered. This may include hospital settings, community inpatient day facilities or rehabilitation facilities where individuals receive treatment overnight. Outpatient settings and communitybased service settings will be excluded.

\section{Types of sources}

This scoping review will include both experimental and quasi-experimental study designs including randomised controlled trials, non-randomised controlled trials, pre-post studies and interrupted time-series studies. In addition, observational studies including prospective and retrospective cohort studies, case-control studies, cross-sectional studies, case series and individual case 
reports will be considered for inclusion. Qualitative and mixed-methods studies will also be considered for inclusion. Grey literature sources such as policy documents and organisational reports will be included. To the best of our knowledge, evidence syntheses which address our research questions do not exist and so cannot be included in this review. However, the reference lists of any evidence syntheses identified by our search will be reviewed for relevant articles. Text and opinion papers will be excluded.

\section{Search strategy}

In collaboration with a JBI-trained information specialist, a search strategy will be developed to locate published articles in peer-reviewed journals and grey literature repositories. An initial limited search of MEDLINE All (Ovid) was undertaken to identify articles on the topic. The text words contained in the titles and abstracts of relevant articles, and the index terms used to describe the articles were used to develop a full search strategy for MEDLINE (online supplemental table 1). The MEDLINE search strategy was peer-reviewed by an information specialist before being translated to Embase (Elsevier Embase. com), CINAHL with Full Text (EBSCOhost), PsycINFO (EBSCOhost) and Scopus (Elsevier Scopus.com) (online supplemental table 1). The reference lists of articles selected for full-text review will be screened for additional papers. Articles published in English, or those available for English translation will be included. No limits will be placed on date of publication.

\section{Information sources}

The databases to be searched include MEDLINE All (Ovid), Embase (Elsevier Embase.com), CINAHL with Full Text (EBSCOhost), PsycINFO (EBSCOhost) and SCOPUS (Elsevier Scopus.com). Grey literature sources will be retrieved and included, following the systematic approach to grey literature searching outlined by Godin et $a .^{23}$ We will not search pre-print servers, as this is not a rapidly emerging topic. However, pre-prints will be screened and considered for inclusion. This topic is not appropriate for clinical trial research; therefore, we will not search trial registries.

\section{Study/Source of evidence selection}

Following the search, all identified records will be collated and uploaded into Covidence systematic review software (Veritas Health Innovation, Melbourne, Australia) and duplicates removed. Following a pilot test, titles and abstracts will be screened by two independent reviewers for assessment against the inclusion criteria. Potentially relevant papers will be retrieved, and full texts will be assessed against the inclusion criteria by two independent reviewers. Reasons for exclusion of full-text papers will be recorded. Any disagreements that arise between reviewers at each stage of the screening process will be resolved through discussion or with an additional reviewer/s. The results of the search will be reported and presented following the Preferred Reporting Items for Systematic Reviews and Meta-analyses for Scoping Reviews. ${ }^{24}$

\section{Data extraction}

Data will be extracted from papers that meet inclusion criteria by two independent reviewers using a standardised data extraction tool (online supplemental table 2). The extracted data will include characteristics of study participants, setting, study design and research objective. Harm reduction strategies employed by each study, barriers and enablers to implementation and patient and health outcome measures will be extracted.

The data extraction tool will be modified and revised as necessary during the process of extracting data from each included paper. Modifications will be detailed in the final scoping review. Authors of papers will be contacted to request missing or additional data, where required. Although scoping review methodology does not require critical appraisal of studies, on request from the knowledge user, it was decided that included published peerreviewed papers and guidelines will be appraised using the Mixed Methods Appraisal Tool. ${ }^{25}$

\section{Data analysis and presentation}

Extracted data will be synthesised and reported in tables throughout the report. A summary of each paper, including setting, publication date and country will be presented to provide context for the research findings. Outcome measures will be reported separately and will be categorised based on harm reduction strategy and barriers and enablers to implementation.

The planned timeline for this review is to complete the title and abstract screening by end of September 2021, have full-text screening completed by mid-October 2021, have data extraction completed by end of October, have a report finalised for submission to the knowledge user by mid-November 2021, and a manuscript finalised and submitted for publication by end of November 2021.

\section{Patient and public involvement}

Two patient partners were consulted during the development of the research questions and inclusion/exclusion criteria and provided feedback of the protocol manuscript. Both patient partners will be engaged to coproduce key messages during the results stage and help in the production of a plain language summary, which will be distributed to local community organisations.

\section{Ethics and dissemination}

Given that this scoping review involves collecting and analysing previously published literature and does not involve research on humans or animals, we did not seek ethics approval from a research ethics board. However, during the initial development of the research question and search strategy, all authors completed an equity, diversity and inclusion reflection activity to identify and reflect on individual bias and position in the literature. It was determined that individuals with lived experience of 
the topic area would be involved throughout the entire study process, from conceptualization to dissemination.

Results of this scoping review will be disseminated to knowledge users and relevant community stakeholders through an initial report. This document will provide a detailed reporting of context, methodology, findings as well as a lay summary, developed in partnership with our patient partners. Additionally, this review will be submitted as a manuscript for publication to a relevant scientific journal.

Acknowledgements We would like to thank our patient partners for their work on this project. We would like to acknowledge the Nova Scotia Health Authority, IWK Health, St Michael's Hospital and the Strategy for Patient-Oriented Research Evidence Alliance for their support of this project.

Contributors JAC, MS, LB and AC conceptualised the study, designed the search strategy and contributed to writing the manuscript. DC and CJohn contributed to writing the manuscript. SM, LW, DS and AER supported the study conceptualisation and informed the search strategy. CJose and MJ provided a patient perspective during conceptualisation of the study and edited the manuscript.

Funding This project is funded by the Strategy for Patient-Oriented Research Evidence Alliance in partnership with the Canadian Institutes of Health Research (Grant number: Q80-21).

Competing interests None declared.

Patient consent for publication Not required.

Provenance and peer review Not commissioned; externally peer reviewed.

Supplemental material This content has been supplied by the author(s). It has not been vetted by BMJ Publishing Group Limited (BMJ) and may not have been peer-reviewed. Any opinions or recommendations discussed are solely those of the author(s) and are not endorsed by BMJ. BMJ disclaims all liability and responsibility arising from any reliance placed on the content. Where the content includes any translated material, BMJ does not warrant the accuracy and reliability of the translations (including but not limited to local regulations, clinical guidelines, terminology, drug names and drug dosages), and is not responsible for any error and/or omissions arising from translation and adaptation or otherwise.

Open access This is an open access article distributed in accordance with the Creative Commons Attribution Non Commercial (CC BY-NC 4.0) license, which permits others to distribute, remix, adapt, build upon this work non-commercially, and license their derivative works on different terms, provided the original work is properly cited, appropriate credit is given, any changes made indicated, and the use is non-commercial. See: http://creativecommons.org/licenses/by-nc/4.0/.

\section{ORCID iDs}

Janet A Curran http://orcid.org/0000-0001-9977-0467

Daniel Crowther http://orcid.org/0000-0002-3402-8189

Catie Johnson http://orcid.org/0000-0001-7956-7226

\section{REFERENCES}

1 McNeil R, Kerr T, Pauly B, et al. Advancing patient-centered care for structurally vulnerable drug-using populations: a qualitative study of the perspectives of people who use drugs regarding the potential integration of harm reduction interventions into hospitals. Addiction 2016;111:685-94.

2 Rachlis BS, Kerr T, Montaner JSG, et al. Harm reduction in hospitals: is it time? Harm Reduct $J$ 2009;6:1-4.

3 Carusone SC, Guta A, Robinson S. "Maybe if I stop the drugs, then maybe they'd care?"-hospital care experiences of people who use drugs. Harm Reduct J 2019;16:1-10.
4 White SR, Bird SM, Merrall ELC, et al. Drugs-Related death soon after hospital-discharge among drug treatment clients in Scotland: record linkage, validation, and investigation of risk-factors. PLoS One 2015;10:e0141073.

5 Hawk M, Coulter RWS, Egan JE, et al. Harm reduction principles for healthcare settings. Harm Reduct $J$ 2017;14:70 http://dx.doi.org. ezproxy.library.dal.ca/

6 Vearrier $\mathrm{L}$. The value of harm reduction for injection drug use: a clinical and public health ethics analysis. Dis Mon 2019;65:119-41.

7 Harm Reduction. The homeless hub, 2021. Available: https://www. homelesshub.ca/about-homelessness/substance-use-addiction/ harm-reduction [Accessed 03 May 2021].

8 Harm reduction, 2021. Available: https://ontario.cmha.ca/harmreduction/ [Accessed 03 May 2021].

9 Canada $\mathrm{H}$. Harm reduction: Canadian drugs and substances strategy. AEM, 2017. Available: https://www.canada.ca/en/healthcanada/services/substance-use/canadian-drugs-substancesstrategy/harm-reduction.html [Accessed 03 May 2021].

10 Platt L, Minozzi S, Reed J, et al. Needle and syringe programmes and opioid substitution therapy for preventing HCV transmission among people who inject drugs: findings from a cochrane review and meta-analysis. Addiction 2018;113:545-63.

11 Csete J, Elliott R. Consumer protection in drug policy: the human rights case for safe supply as an element of harm reduction. Int $J$ Drug Policy 2021;91:102976.

12 Olding M, Ivsins A, Mayer S, et al. A low-barrier and comprehensive community-based harm-reduction site in Vancouver, Canada. Am J Public Health 2020;110:833-5 http://dx.doi.org.ezproxy.library.dal.ca/

13 Courser MW, Raffle H. With crisis comes opportunity: unanticipated benefits resulting from pivots to take-home naloxone (THN) programs during the COVID-19 pandemic. J Subst Abuse Treat 2021;122:108220.

14 Sharma M, Lamba W, Cauderella A, et al. Harm reduction in hospitals. Harm Reduct J 2017;14:1-4.

15 Elphinston RA, Sterling M, Leung J, et al. Brief psychological interventions for reducing prescription opioid use, related harm, and pain intensity in patients with chronic pain: a systematic review and assessment of patient interest. Clin J Pain 2021;37:270-80.

16 Sharpe S, Kool B, Whittaker R, et al. Effect of a text message intervention on alcohol-related harms and behaviours: secondary outcomes of a randomised controlled trial. BMC Res Notes 2019;12:267.

17 Saunders E, Metcalf SA, Walsh O, et al. "You can see those concentric rings going out": emergency personnel's experiences treating overdose and perspectives on policy-level responses to the opioid crisis in New Hampshire. Drug Alcohol Depend 2019;204:107555.

18 Calcaterra SL, Binswanger IA, Edelman EJ, et al. The impact of access to addiction specialist on attitudes, beliefs and hospitalbased opioid use disorder related care: a survey of hospitalist physicians. Subst Abus 2020;0:1-9.

19 Strike C, Robinson S, Guta A, et al. Illicit drug use while admitted to hospital: patient and health care provider perspectives. PLoS One 2020;15:e0229713 http://dx.doi.org.ezproxy.library.dal.ca/

20 Hobden KL, Cunningham JA. Barriers to the dissemination of four harm reduction strategies: a survey of addiction treatment providers in Ontario. Harm Reduct J 2006;3:35-20.

21 Aromataris E, Munn Z. JBI manual for evidence synthesis. JBI, 2020.

22 Government of Canada Cl of HR. Guide to knowledge translation planning at CIHR: integrated and End-of-Grant approaches, 2012. Available: https://cihr-irsc.gc.ca/e/45321.html [Accessed $07 \mathrm{Jul}$ 2021].

23 Godin K, Stapleton J, Kirkpatrick SI, et al. Applying systematic review search methods to the grey literature: a case study examining guidelines for school-based breakfast programs in Canada. Syst Rev 2015;4:138.

24 Tricco AC, Lillie E, Zarin W, et al. PRISMA extension for scoping reviews (PRISMA-ScR): checklist and explanation. Ann Intern Med 2018;169:467-73.

25 Hong QN, Pluye P, Fabregues S. Mixed methods appraisal tool (mmat) version 2018: user guide, 2018. Available: http://mixedmet hodsappraisaltoolpublic.pbworks.com/w/file/fetch/127916259/ MMAT_2018_criteria-manual_2018-08-01_ENG.pdf [Accessed 05 Jul 2021]. 